\title{
Salmonella persisters undermine host immune defenses during antibiotic treatment
}

\author{
Daphne A. C. Stapels ${ }^{1 *} \dagger$, Peter W. S. Hill ${ }^{1 *}$, Alexander J. Westermann ${ }^{2,3}$, \\ Robert A. Fisher' ${ }^{1}$, Teresa L. Thurston ${ }^{1}$, Antoine-Emmanuel Saliba ${ }^{3}$, \\ Isabelle Blommestein ${ }^{1}$, Jörg Vogel ${ }^{2,3}$, Sophie Helaine ${ }_{\ddagger} \neq$
}

Many bacterial infections are hard to treat and tend to relapse, possibly due to the presence of antibiotic-tolerant persisters. In vitro, persister cells appear to be dormant. After uptake of Salmonella species by macrophages, nongrowing persisters also occur, but their physiological state is poorly understood. In this work, we show that Salmonella persisters arising during macrophage infection maintain a metabolically active state. Persisters reprogram macrophages by means of effectors secreted by the Salmonella pathogenicity island 2 type 3 secretion system. These effectors dampened proinflammatory innate immune responses and induced anti-inflammatory macrophage polarization. Such reprogramming allowed nongrowing Salmonella cells to survive for extended periods in their host. Persisters undermining host immune defenses might confer an advantage to the pathogen during relapse once antibiotic pressure is relieved.

更 uring growth, genetically clonal bacterial populations contain a small fraction of nongrowing, nondividing cells that arise from transient, reversible, phenotype switching. These growth-arrested cells are usually tolerant to antibiotics and are called antibiotic persisters (1). Previously, we showed that a large proportion of the intracellular pathogen Salmonella enterica serovar Typhimurium (Salmonella Typhimurium) adopts a nongrowing, antibiotic-tolerant state within macrophages (2). We also showed that the first Salmonella persister cells that regrow upon release from their host cells are those that maintain metabolic activity during infection (2). Similar nongrowing but metabolically active bacteria have also been observed in macrophages infected with Mycobacterium tuberculosis (3). By contrast, when Salmonella and other bacterial species are grown in laboratory culture media, persisters are often observed to be inactive (i.e., dormant) (4-6).

To assess whether retention of transcriptional and translational activity might confer an additional physiological benefit to nongrowing bacteria within a host cell, we infected mouse bone marrow-derived macrophages with wild-type Salmonella Typhimurium cells carrying a reporter plasmid that allowed for tracking of bacterial proliferation and activity (7) (figs. S1 and S2). Spontaneous regrowth of nongrowing

${ }^{1}$ MRC Centre for Molecular Bacteriology and Infection, Imperial College London, London, UK. ${ }^{2}$ Institute of Molecular Infection Biology, University of Würzburg, Würzburg. Germany. ${ }^{3}$ Helmholtz Institute for RNA-based Infection Research, Würzburg, Germany.

*These authors contributed equally to this work. †Present address: Department of Medical Microbiology. University Medical Center Utrecht, Utrecht, Netherlands.

‡Corresponding author. Email: s.helaine@imperial.ac.uk
Salmonella cells following infection and antibiotic treatment arose exclusively from active rather than inactive bacteria; the latter failed to regrow even after days of incubation (Fig. 1A). In addition, we artificially generated a population of nongrowing and translation-incompetent Salmonella cells through exposure to bacteriostatic concentrations of chloramphenicol (fig. S3) and subsequently monitored survival of these dormant bacteria after exposure to the bactericidal antibiotic cefotaxime. Although the inactive Salmonella cells withstood exposure to cefotaxime in laboratory medium, they did not survive within macrophages cultured with antibiotics (Fig. 1A).

After entry into macrophages, Salmonella Typhimurium induces expression of the Salmonella pathogenicity island 2 (SPI-2) type 3 secretion system (T3SS), through which it translocates $\sim 30$ effectors that inhibit host cell processes that are detrimental to the pathogen $(8,9)$. We hypothesized that as well as maintaining transcriptional and translational activity, persisters also translocate SPI-2 effectors. We used the SPI-2 $s s a G$ promoter fused to unstable enhanced green fluorescent protein (eGFP) (10) to test for SPI-2 gene expression in single cells. $s s a G$ promoter expression was observed in nongrowing bacteria that retained transcriptional and translational activity (Fig. 1B). Furthermore, Salmonella effector proteins were detected in the host cell cytosol via Western blotting of macrophages containing pure populations of growing or persister

To understand how persisters shape their host environment, we used dual RNA sequencing (RNA-seq) (11) on infected macrophage subpopulations to analyze host and pathogen transcriptomes simultaneously (12) (fig. S5). The cells (Fig. 1C and fig. S4). majority of dual RNA-seq reads from infected macrophages aligned to the host genome, with relative amounts of bacterial reads being proportional to the average number of bacteria present per cell (fig. S5D and table S1).

Principal component analysis (fig. S6A) and clustering analysis (Fig. 2A and table S2) on the transcriptome profiles of all macrophage populations confirmed the expected general differences between challenged and naïve macrophages (Fig. 2A, clusters I and II, and fig. S6A). Among challenged macrophages, the greatest differences occurred between macrophages containing viable bacteria (growing or nongrowing) and macrophages that had killed the bacteria they had engulfed [host killed (HK)] or bystander macrophages. Clustering analysis pinpointed two large groups of genes (Fig. 2A, clusters III and IV) responsible for this separation (Fig. 2B). Of these, members of cluster III were enriched with genes involved in classical, proinflammatory macrophage activation (i.e.,. M1), and cluster IV was enriched with genes associated with alternative, anti-inflammatory (i.e., M2) macrophage activation. Inspection of the dual RNA-seq data for five representative M1 activation markers ( $N f k b 2, C d 40, \Pi l 1 b, N l r p 3$, and Tnf) (fig. S6B, left panel) confirmed that their up-regulation during infection was dampened in macrophages containing viable bacteria. In contrast, five M2 activation markers (Il4ra, Arg1, OdcI, Ppard, and TimpI) (fig. S6B, right panel) were upregulated in macrophages containing viable bacteria. There was a significant overlap of these gene clusters with our previously defined M1 gene set $\left(P=5.2 \times 10^{-19}\right.$, hypergeometric test $)$ and $\mathrm{M} 2$ gene set $\left(P=1.2 \times 10^{-38}\right.$, hypergeometric test) (Fig. 2C), deduced from singlecell RNA-seq data (13). As clusters III and IV contain considerably more genes, we consider them more complete M1 and M2 polarization gene sets.

Principal component analysis (fig. S6A) and clustering analysis of bacterial genes in the dual RNA-seq dataset (fig. S6C and table S3) showed expected differences, such as expression of flagellar genes in the inoculum. By comparison, intracellular bacteria showed elevated expression of infection-associated genes (14). Notably, the transcriptomes of intracellularly growing and nongrowing bacteria were similar (fig. S6, A and C), and both expressed genes encoding the SPI-2 T3SS apparatus and its translocated effectors (fig. S6D).

We performed interspecies expression correlation analysis on the dual RNA-seq data to reveal the host consequences of bacterial SPI-2 T3SS expression. Of the 4817 defined murine gene sets tested, SPI-2 T3SS gene expression showed the strongest positive correlation with M2 antiinflammatory cluster IV genes and the strongest negative correlation with $\mathrm{M} 1$ proinflammatory cluster III genes (Fig. 2, D and E, and table S4). Of the Salmonella regulons, only $\mathrm{PhoP} / \mathrm{Q}$ and SPI-2 T3SS showed this (anti-)correlation pattern with the M1 and M2 host gene sets (table S5 and fig. S6, F and G). Because PhoP/Q is required for SPI-2 T3SS activation (15), the data suggest 


\section{A}

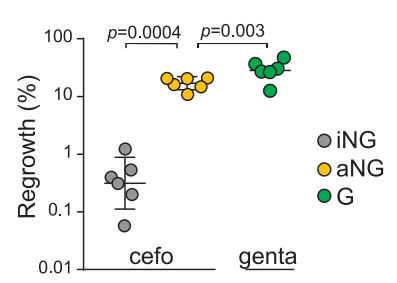

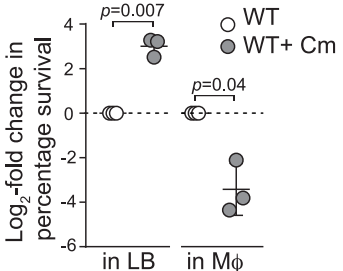

Fig. 1. Salmonella antibiotic persisters during macrophage infection are metabolically active, transcribing, translating, and translocating SPI-2 T3SS effectors. (A) (Left) Regrowth on laboratory medium of sorted inactive nongrowing (iNG), active nongrowing (aNG), or growing $(\mathrm{G})$ bacteria after 24 hours of infection under exposure to cefotaxime (cefo) or gentamicin (genta), respectively. (Right) Survival of translationally blocked nongrowing Salmonella subsequently exposed to bactericidal concentrations of cefotaxime in laboratory medium (LB) or macrophages (M $\phi)$ for 24 hours. $P$ values are indicated (unpaired $t$ test for aNG versus G; paired $t$ test for other comparisons; tests on the log-transformed data). Error bars depict means and SD. (B) Expression of unstable eGFP controlled by a SPI-2 promoter (PssaG) in intracellular bacteria at 24 hours postuptake depends on the growth and activity status. A representative histogram is shown on the left, and quantification of results from six independent repeats is shown on the right. $P$ values are indicated (paired $t$ test); error bars depict means and SD. (C) Translocation of hemagglutinin (HA)-tagged SPI-2 T3SS effectors detected in the bacterial pellet or host cell cytosol after lysis of bystander (Byst) M $\phi$ or M $\phi$ containing growing (G) or nongrowing (NG) Salmonella Typhimurium or a secretion-deficient (ssaV) mutant 20 hours after uptake. * detected effector.

Fig. 2. Dual RNA-seq implicates SPI-2 in dampening M1 and promoting M2 macrophage polarization. (A) Clustering analysis of host genes differentially expressed between any two subpopulations, with selected enriched terms. Analyzed subpopulations of M $\phi$ were naïve, bystander, or contained hostkilled (HK), nongrowing (NG), or growing $(G)$ bacteria. (B) Principal components analysis of $\mathrm{M} \phi$ transcriptomes based on M1 (cluster III in panel A) and M2 (cluster IV in panel A) polarization genes. The subsets of $\mathrm{M} \phi$ are color coded, and the three biological repeats are indicated with different symbols. (C) Venn diagram showing the overlap between M1 (top) and M2 (bottom) polarization genes identified in (13) and extended $\mathrm{M} 1$ and $\mathrm{M} 2$ genes identified here (clusters III and IV). Numbers of genes within each gene set are indicated. (D) Interspecies correlation analysis between SPI-2 apparatus and effector gene

expression patterns in Salmonella and gene expression patterns of defined host gene sets in infected dual RNA-seq samples. Plot of the gene set enrichment analysis (GSEA) score ( $x$ axis) and - $\log _{10}$ family-wise error rate (FWER)-adjusted $P$ value ( $y$ axis), based on correlations between $z$-scorenormalized host gene expression and average $z$-score-normalized SPI-2 apparatus and effector expression for all 4817 tested murine gene sets.

(E) Boxplots with boxes (the median upper and lower quartiles) and whiskers (the lowest and highest values) depicting the distribution of correlations of interesting gene sets with SPI-2 genes (FWER-adjusted $P$ values are indicated)
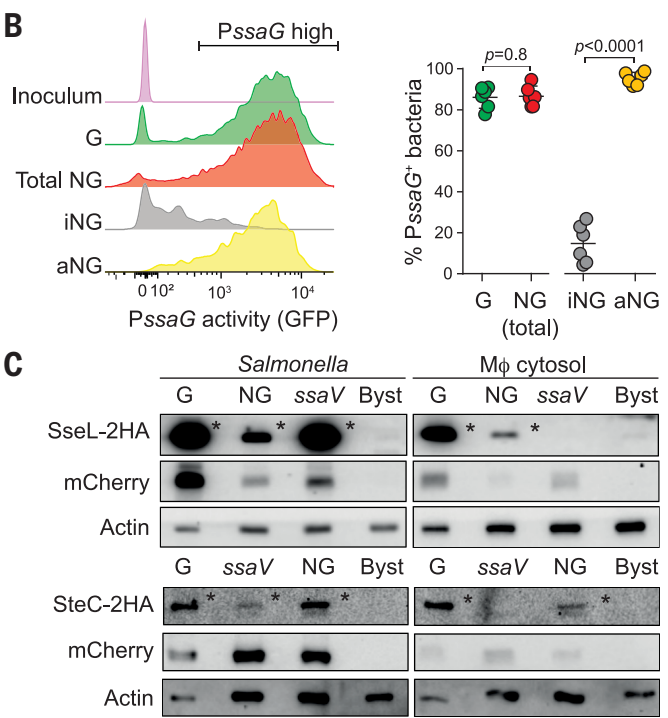

B Polarization gene expression

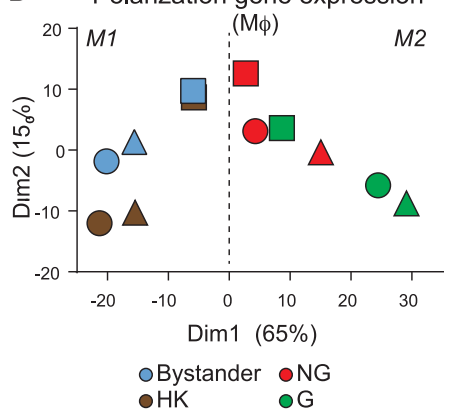

E

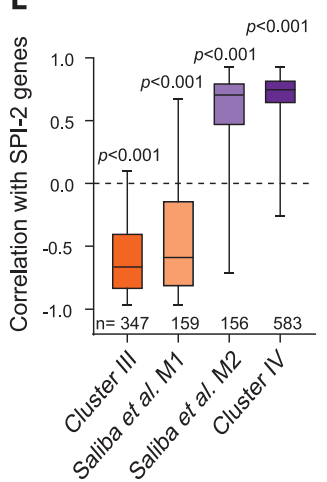


A

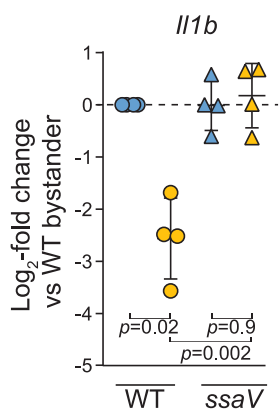

Nfkb2

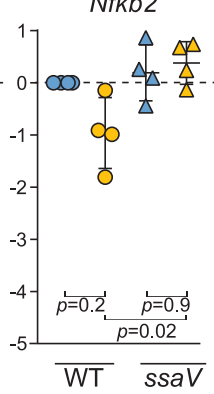

Cd40

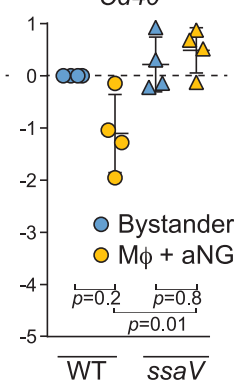

B

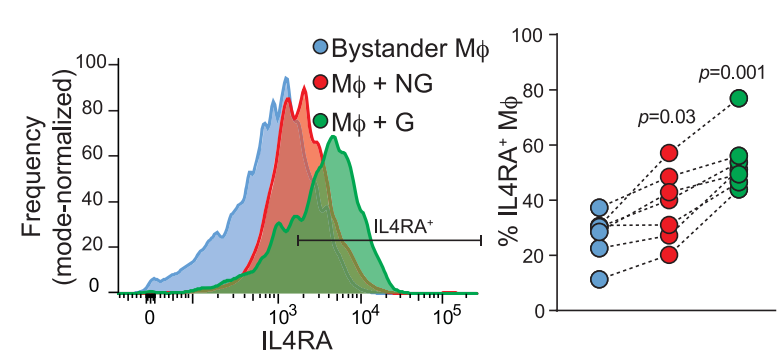

C

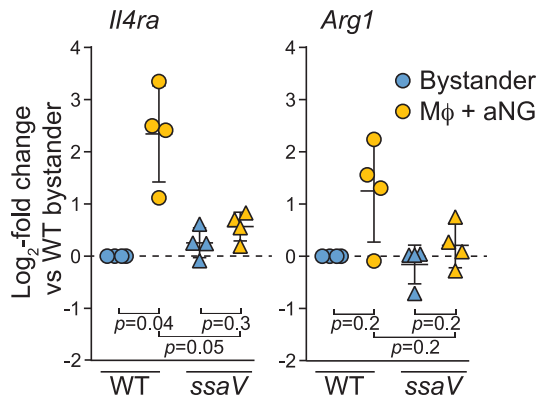

$\mathbf{E}$

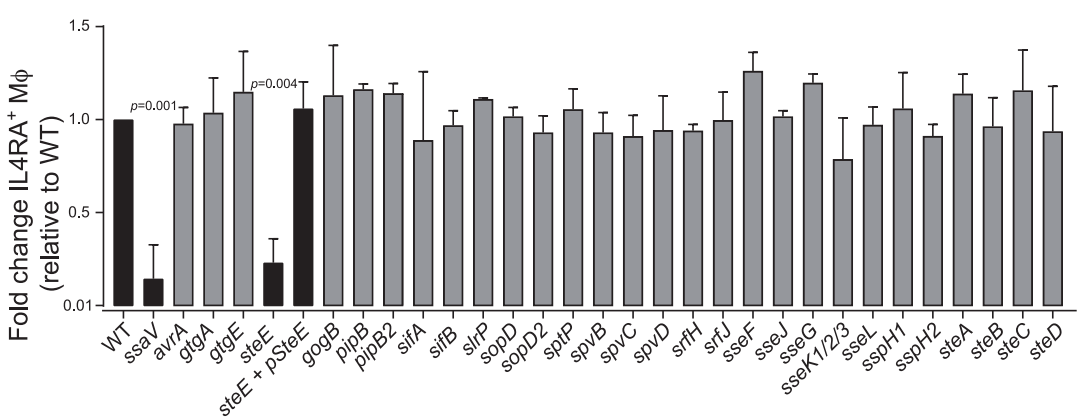

D

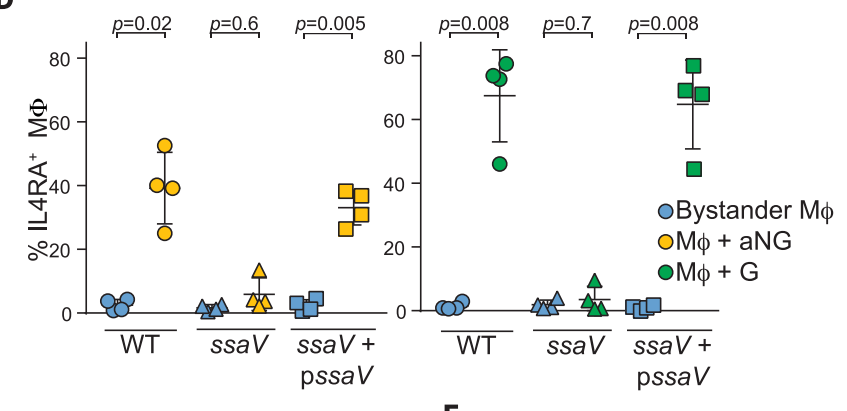

Fig. 3. Growing and nongrowing Salmonella use SPI-2 to dampen M1 and drive M2 macrophage polarization.
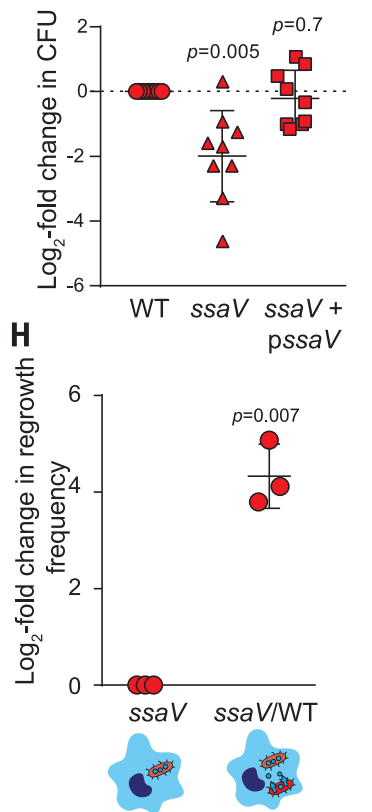

(A) mRNA levels of M1 genes upon infection by wild-type (WT) or ssaV mutant active nongrowing Salmonella 18 hours after uptake (cefotaxime treated). Relative expression levels were measured via qRT-PCR and calculated by using the cycle threshold $\left(\Delta \Delta C_{T}\right)$ method (expression levels relative to those of control DsRed RNA and bystander $M \phi$ ) [paired analysis of variance (ANOVA) with multiple testing among shown groups; adjusted $P$ values are indicated; error bars depict means and SD]. (B) Representative histograms of IL4RA expressed by splenic M $\phi$ from one mouse (left) and the proportion of IL4RA-positive M $\phi$ in multiple mice (right). Subpopulations of $M \phi$ from the same mouse are connected with a dotted line. (Paired ANOVA, multiple testing against bystander M $\phi$; adjusted $P$ values are indicated). (C) Expression levels of M2 polarization genes, determined as described for (A). (D) Proportion of IL4RA-positive infected M $\mathrm{M} 24$ hours after uptake of WT, ssaV mutant, or ssaV-complemented bacteria. Either M $\phi$ containing active nongrowing ( $\mathrm{aNG}$ ) bacteria (cefotaxime treated; left) or $\mathrm{M \phi}$ containing a similar amount of growing (G) bacteria for each strain (right) were analyzed (paired ANOVA with multiple testing against corresponding bystander M $\phi$; adjusted $P$ values are indicated; error bars depict means and SD). (E) Screen of SPI-2 effector mutants for accumulation of IL4RA in M $\phi$ infected with growing bacteria 24 hours after uptake [unpaired ANOVA with multiple testing (Dunnett's test) against corresponding $M \phi$ infected with WT Salmonella]. Adjusted $P$ values are indicated; error bars depict SD. (F) IL4RA expression in bystander M $\phi$ and M $\phi$ with HK, NG, or G Salmonella continuously exposed to IFN- $\gamma 24$ hours after uptake. Populations of M containing WT or ssaV mutant bacteria were gated to contain similar bacterial loads (paired ANOVA, repeated testing against bystander $\mathrm{M} \phi$; adjusted $P$ values are indicated; error bars depict means and SD). (G) Intramacrophage long-term survival of NG Salmonella after 48 hours cefotaxime treatment, corrected for cytotoxicity (paired ANOVA with multiple testing against WT; adjusted $P$ values are indicated; error bars depict means and SD). (H) Intramacrophage survival of NG ssaV mutant Salmonella after 48 hours of cefotaxime treatment, following sorting of $M_{\phi}$ singly infected with mCherry-expressing ssaV mutant or $M \phi$ infected with a mixed population of mCherry-expressing ssaV mutant and GFP-expressing WT bacteria (unpaired $t$ test against single infection; $P$ values are indicated; error bars depict means and SD). 


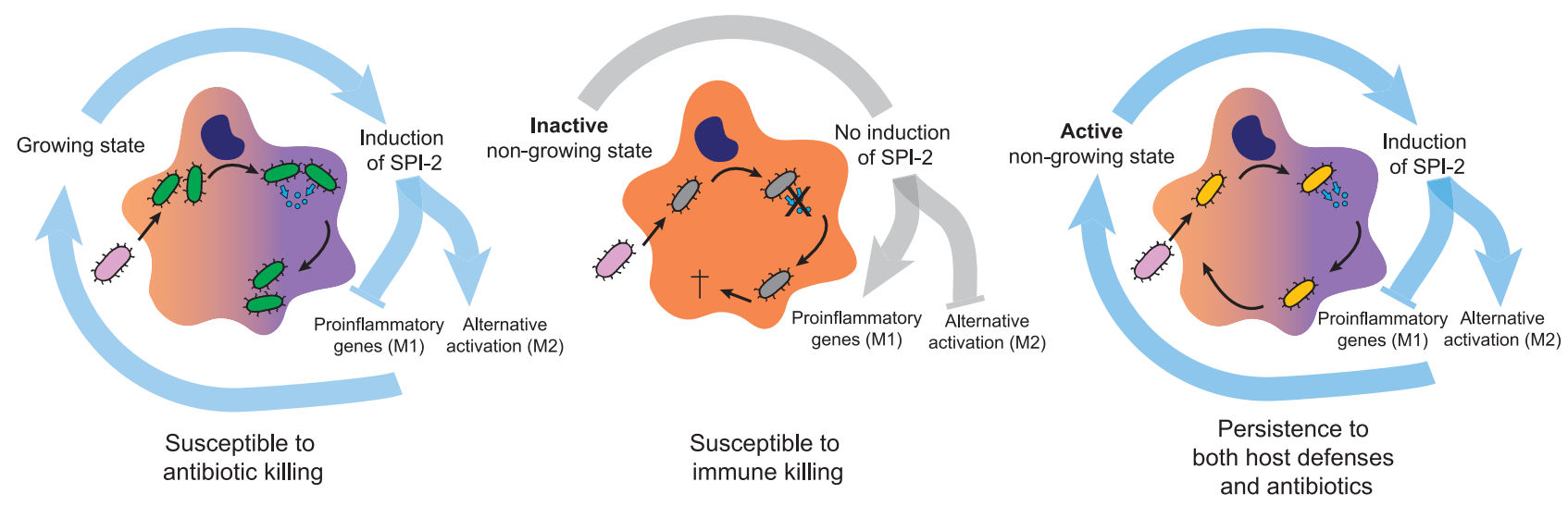

Fig. 4. Persisters undermine the host innate immune response and enable long-term survival. Model of macrophage manipulation by Salmonella persisters. Growing Salmonella bacteria translocate SPI-2 effectors that manipulate host cell polarization and create a less hostile environment. Proliferation makes the bacteria susceptible to antibiotic killing (left). Inactive nongrowing Salmonella cannot translocate SPI-2 effectors and are killed in the strongly antimicrobial environment (middle). Active persisters manipulate host cell polarization through translocation of SPI-2 effectors, and in turn they retain their activity and maintain the ability to survive in the host while being antibiotic tolerant (right).

that SPI-2 T3SS effectors modulate M1 and M2 polarization.

Macrophages containing active nongrowing Salmonella displayed an intermediate expression profile between the M1 and M2 phenotypes (Fig. 2B). Previous single-cell RNA-seq data led us to conclude that nongrowing bacteria inhabit M1-like cells whereas growing Salmonella associate with M2-like macrophages (13). Reanalysis of our previous single-cell RNA-seq data (13) with the extended M1 and M2 gene sets showed there was a bimodality of M1 and M2 gene expression in macrophages containing nongrowing bacteria (fig. S7A). By contrast, bystander macrophages had an M1 bias, and those containing growing bacteria had a clear M2 bias (fig. S7a). The levels of the macrophage surface M2 marker IL4RA (the alpha-subunit of the interleukin-4 receptor) were significantly increased in macrophages containing nongrowing active Salmonella compared with macrophages containing nongrowing inactive or host-killed bacteria (fig. S7B). Therefore, the intermediate population-level M1 and M2 expression profile of the host cell probably reflects an underlying heterogeneity of transcriptional and translational activity and also SPI-2 expression in nongrowing intramacrophage Salmonella (Fig. 1) (2).

The correlation between SPI-2 effector expression and macrophage polarization could be explained by the presence of subsets of macrophages prior to infection with depressed inflammatory responses and/or increased permissiveness. Alternatively, this could be a direct result of activity of SPI-2 effectors, some of which are known to downregulate proinflammatory responses, such as NF- $\kappa$ B activation (9). Consistent with the latter, when we compared the levels of three mRNAs for established M1 markers (Cd40, Il1b, and $N f k b 2$ ) between macrophages containing active nongrowing wild-type or SPI-2-null ( $s s a \mathrm{~V}$ mutant) bacteria via reverse transcription quantitative polymerase chain reaction (RT-qPCR), wild-type Salmonella was associated with down- regulated expression of the three markers during exposure to antibiotics, whereas cells infected with the SPI-2-null mutant bacteria were not (Fig. 3A). Therefore, active nongrowing bacteria use SPI-2 T3SS effectors to counteract macrophage M1 activation (9) and are able to do so despite sustained exposure to the proinflammatory interferon gamma (IFN- $\gamma$ ) cytokine (fig. S8, A and B)

In addition, analysis of the dual RNA-seq data and our previous single-cell RNA-seq data (13) suggested that Salmonella Typhimurium actively promotes macrophage M2 polarization. In a murine model of long-term infection, Salmonella Typhimurium resided in M2 macrophages (16) rather than in proinflammatory $\mathrm{M} 1$ macrophages (17-19), suggesting that the conditions within M2 macrophages enable prolonged bacterial survival. Consistent with this conclusion, in a mouse model of acute systemic infection (fig. S8C) the M2 marker IL4RA was highly expressed by splenic macrophages isolated 3 days after intraperitoneal Salmonella Typhimurium injection, with the majority of the growing bacteria inside the macrophages showing high ILARA expression (Fig. 3B). There was greater heterogeneity in IL4RA expression within the macrophage population containing nongrowing bacteria (Fig. 3B). Despite exposure to antibiotics, active nongrowing wild-type Salmonella cells, but not SPI-2null mutant cells, showed up-regulated expression of the two M2 markers tested in macrophages (Fig. 3C).

Further investigation revealed that during infection with a SPI-2-null strain, accumulation of IL4RA was abrogated in all macrophages infected with viable bacteria regardless of the bacterial growth state (Fig. 3D and fig. S8D) (20). The M2-like polarization profile induced by Salmonella was recapitulated best by exposure to both IL-4 and IL-10 (fig. S9). Screening a collection of all SPI-2 effector mutants revealed that M2-like polarization is driven solely by the SteE (also known as SarA) effector (Fig. 3F and fig. S8F); a recent report showed that SteE promotes secretion of IL-10 from infected B cells (21). Although an steE mutant no longer triggered M2-like polarization of host cells, the mutant still dampened the M1 response (figs. S8, E and F, and S10), suggesting that M1 suppression and M2 polarization are independent of each other. Nongrowing intracellular Salmonella can thus express and translocate sufficient quantities of effectors to cause major changes in the immune status of the infected host cell, even during exposure to antibiotics and IFN- $\gamma$.

Finally, after 48 hours of antibiotic exposure within macrophages, nongrowing mutant bacteria lacking a functional SPI-2 apparatus showed significantly reduced survival compared with their wild-type counterparts (Fig. 3G). However, in mixed strain infections we found that wild-type persisters rescued survival of SPI-2-null persisters in coinfected cells (Fig. $3 \mathrm{H}$ ), illustrating that the decreased survival of SPI-2-null persisters was exclusively a consequence of a failure to manipulate the intracellular host environment in which they resided.

Collectively, our data show that after infection of macrophages, maintenance of transcriptional and translational activity enables nongrowing Salmonella Typhimurium to translocate SPI-2 T3SS effectors into the host cell. Thus, unlike bacterial persisters in laboratory medium, which apparently become dormant $(2,4-6)$, intracellular Salmonella cells maintain effector delivery but cease to grow. This strategy enables a subpopulation of intracellular bacteria to survive antibiotic exposure and reprogram their macrophage host cell to promote long-term bacterial survival (Fig. 4). Reprogramming of the host cell by Salmonella not only suppresses M1 bactericidal responses (9) but also increases permissiveness of the now-M2-biased cells for the pathogen, potentially by modulating host cell metabolism $(17,22)$. We showed that the capacity of Salmonella to direct macrophage M2 polarization is driven by the SPI-2 T3SS effector SteE. 
Similarly, M. tuberculosis infections have been accompanied by an expansion of a population of permissive macrophages (23-27), raising the possibility that mycobacteria and other intracellular pathogens employ similar mechanisms for modulating host cell polarization. Pathogen manipulation of host cells is accompanied by changes in the repertoire of secreted cytokines (21). Rather than being dormant, nongrowing persister cells of an intracellular bacterial pathogen are able to subvert host immune defenses, even under antibiotic treatment, potentially making the host environment more permissive for recrudescent infection.

\section{REFERENCES AND NOTES}

1. R. A. Fisher, B. Gollan, S. Helaine, Nat. Rev. Microbiol. 15 453-464 (2017)

2. S. Helaine et al., Science 343, 204-208 (2014).

3. G. Manina, N. Dhar, J. D. McKinney, Cell Host Microbe 17 32-46 (2015).

. E. Maisonneuve, K. Gerdes, Cell 157, 539-548 (2014).

5. Y. Shan et al., mBio 8, e02267-16 (2017).

6. B. P. Conlon et al., Nat. Microbiol. 1, 16051 (2016).

7. S. Helaine et al., Proc. Natl. Acad. Sci. U.S.A. 107, 3746-3751 (2010).

K. McGourty et al., Science 338, 963-967 (2012)

E Jennings, T. L. Thurston, D. W. Holden, Cell Host Microbe 22, 217-231 (2017).
10. J. A. Ibarra et al., Microbiology 156, 1120-1133 (2010).

1. A. J. Westermann et al., Nature 529, 496-501 (2016).

12. A. J. Westermann, L. Barquist, J. Vogel, PLOS Pathog. 13, e1006033 (2017).

13. A.-E. Saliba et al., Nat. Microbiol. 2, 16206 (2016).

14. S. Eriksson, S. Lucchini, A. Thompson, M. Rhen, J. C. D. Hinton, Mol. Microbiol. 47, 103-118 (2003).

15. E. Fass, E. A. Groisman, Curr. Opin. Microbiol. 12, 199-204 (2009).

16. P. J. Murray et al., Immunity 41, 14-20 (2014).

17. N. A. Eisele et al., Cell Host Microbe 14, 171-182 (2013).

18. M. W. McCoy, S. M. Moreland, C. S. Detweiler, Infect. Immun. 80, 3642-3649 (2012)

19. R. N. Nix, S. E. Altschuler, P. M. Henson, C. S. Detweiler, PLOS Pathog. 3, e193 (2007).

20. R. Figueira, K. G. Watson, D. W. Holden, S. Helaine, mBio 4 e00065-13 (2013)

21. S. L. Jaslow et al., Cell Reports 23, 3525-3536 (2018).

22. J. Van den Bossche, L. A. O'Neill, D. Menon, Trends Immunol. 38, 395-406 (2017)

23. D. M. Hardbower et al., Proc. Natl. Acad. Sci. U.S.A. 114 E751-E760 (2017).

24. F. O'Rourke et al., Cell. Microbiol. 17, 1447-1463 (2015).

25. K. A. Shirey, L. E. Cole, A. D. Keegan, S. N. Vogel, J. Immunol. 181, 4159-4167 (2008)

26. M. Benoit, B. Barbarat, A. Bernard, D. Olive, J. L. Mege, Eur. J. Immunol. 38, 1065-1070 (2008).

27. L. Huang, E. V. Nazarova, S. Tan, Y. Liu, D. G. Russell, J. Exp. Med. 215, 1135-1152 (2018).

\section{ACKNOWLEDGMENTS}

We thank members from the Holden and Helaine labs for sharing constructs and protocols and scientific discussions: I. Glegola-Madejska for help with the animal experiments; and D. Holden for careful reading of the manuscript. Funding: This research was supported by an MRC Career Development Award (MR/M009629/1) from the Medical Research Council (UK) and Lister Institute Research Prize 2017 to S.H., an EMBO long-term fellowship (ALTF 441-2015) to D.A.C.S., and an Elite Advancement Ph.D. stipend from the Universität Bayern e.V., Germany, to A.J.W. Author contributions: S.H. and J.V. outlined the study; D.A.C.S., P.W.S.H., A.J.W., R.F., I.B., and S.H. performed experiments; P.W.S.H. conducted the bioinformatic analysis; D.A.C.S., P.W.S.H., A.J.W., R.F., I.B., A.-E.S., and T.L.T. analyzed the data; D.A.C.S., P.W.S.H., A.J.W., and S.H. interpreted data; and D.A.C.S., P.W.S.H., and S.H. wrote the manuscript with contributions of other authors. Competing interests: We declare no competing interests. Data and materials availability: The dual RNA-seq data are summarized in table S1; results of analyses are in tables S2 to S5, S8, and S9; and the data are archived on GEO (accession: GSE104785).

\section{SUPPLEMENTARY MATERIALS}

www.sciencemag.org/content/362/6419/1156/suppl/DC Materials and Methods

Figs. S1 to S10

Tables S1 to S9

References (28-32)

28 March 2018; resubmitted 25 July 2018

Accepted 31 October 2018

10.1126/science.aat7148 


\section{Science}

\section{Salmonella persisters undermine host immune defenses during antibiotic treatment}

Daphne A. C. Stapels, Peter W. S. Hill, Alexander J. Westermann, Robert A. Fisher, Teresa L. Thurston, Antoine-Emmanuel Saliba, Isabelle Blommestein, Jörg Vogel and Sophie Helaine

Science 362 (6419), 1156-1160.

DOI: $10.1126 /$ science.aat7148

\section{Actively persistent Salmonella}

A proportion of Salmonella cells can enter a reversible state of growth arrest, which allows them to tolerate environmental stress such as antibiotics. Stapels et al. found that these cells are not dormant but are actively modulating their environment. Salmonella within their host macrophage niche deployed a specialized type 3 secretory system called SPI-2 to deliver virulence factors, including SteE, into host cells. SteE changed the cytokine profile of the infected macrophages to reprogram them into a noninflammatory and infection-permissive state. Thus, when antibiotics were removed, the Salmonella could reemerge and cause disease. Science, this issue p. 1156

ARTICLE TOOLS

REFERENCES

PERMISSIONS http://science.sciencemag.org/content/362/6419/1156

http://science.sciencemag.org/content/suppl/2018/12/05/362.6419.1156.DC1

This article cites 32 articles, 11 of which you can access for free http://science.sciencemag.org/content/362/6419/1156\#BIBL

http://www.sciencemag.org/help/reprints-and-permissions

Science (print ISSN 0036-8075; online ISSN 1095-9203) is published by the American Association for the Advancement of Science, 1200 New York Avenue NW, Washington, DC 20005. 2017 (C) The Authors, some rights reserved; exclusive licensee American Association for the Advancement of Science. No claim to original U.S. Government Works. The title Science is a registered trademark of AAAS. 